\title{
Time-Step Volume-Preserving Control of Chemical Reactors
}

\author{
José-Manuel Zaldívar Comenges ${ }^{*}, 1$ and Fernanda Strozzi ${ }^{2}$ \\ ${ }^{I}$ European Commission, DG-Joint Research Centre, Institute for Environment and Sustainability, Italy \\ ${ }^{2}$ Carlo Cattaneo University, Quantitative Methods Institute, Castellanza, Italy
}

\begin{abstract}
We present a general control and optimization strategy based on time-step state space volume control. This is performed by maintaining the divergence of the system close to zero. The on-line implementation is shown for batch chemical reactors and the simulated results are compared with a traditional control scheme.
\end{abstract}

\section{INTRODUCTION}

One of the main problems for chemical reactors carrying out exothermic reactions is the loss of temperature control. However, an excessive careful operation of the reactor, outside their optimal operating conditions, will provoke long reactions batches with the subsequent economic losses. Therefore, for batch processes, the optimal conduct of the process implies to avoid situations in which the rate of heat generation by chemical reaction exceeds the rate of heat removal but to work as close as possible to this point [1-2], whereas for semibatch reactors(SBR), the best operating conditions require to work on quasi instantaneous mode, i.e. with the reaction rate controlled by the dosing rate [3-4]. Safety problems in SBRs may arise when there is accumulation inside the reactor due to low reaction rates as compared with the dosing rate. In this case, the reactor works close to batch conditions.

In a series of previous works [5-8], a new criterion to delimit runaway boundaries was developed applying techniques from nonlinear dynamical systems theory to characterize the sensitivity of chemical reactors. The runaway detection criterion was defined as when the divergence of the reactor becomes positive on a segment of the reaction path, i.e. div $>0$. Furthermore, the on-line application of the divergence criterion was demonstrated for isoperibolic -constant jacket temperature- [9] and isothermal reactors [10] as well as experimentally in a pilot plant [11-12] and in an industrial reactor [13].

If $d i v>0$ implies that we are in a dangerous situation, and div $<0$ implies that we are in non-ignition conditions, i.e. long batch times, it seems logical to define as optimum conditions from safety and performance points of view, as those that produce $d i v=0$ at any given instant. The divergence of a dynamical system is related to the variation of its total energy, which in turn is related with its state space volume. Therefore, $d i v=0$ is equivalent to require state space volume preservation at each time step. In this sense, we are trying to convert a dissipative system, the batch reactor (BR), into a conservative (Hamiltonian) system. By controlling the divergence we have, in a certain sense controlled the changes in the total energy of the system. When there is an increase,

*Address correspondence to this author at the European Commission, DGJoint Research Centre, Institute for Environment and Sustainability, Italy; E-mail: jose.zaldivar-comenges@jrc.it $d i v>0$, or a decrease, $d i v<0$, we will try to decelerate or accelerate the process by increasing or decreasing the jacket temperature.

This approach was tested analytically using a BR in which a first order reaction took place [14]. Therefore, the control system instead of considering the difference between the set-point and the reactor temperatures, $\left(T^{s p}-T_{r}\right)$ when working on isothermal mode or set-point and jacket temperature, $\left(T^{s p}-T_{j}\right)$ when working on isoperibolic mode, considered the divergence (0-div) as goal function for the control. The results for batch simulated experiments showed that this control strategy is able to maintain safe conditions in case where traditional control systems will lead to a runaway, and increase the reaction rate for operating conditions where the batch production time would be too long.

As the divergence of the system can be obtained from temperature measurements using state space reconstruction techniques $[5,12]$, we have developed in this work several control strategies and tested their application on-line using, not the analytical values of the divergence as in [14], but its reconstructed values from temperature measurements. The results show that the reconstructed divergence can be used for control and optimization of chemical reactors. In addition, by maintaining $d i v \approx 0$ we are avoiding dangerous conditions as described above [5].

The next step of this research will be the real implementation of this control strategy on an experimental reactor and the testing of their robustness against noise and operator manipulations that occur in real plants.

\section{METHODS AND APPROACH}

\section{Divergence and State Space Volume}

Let us consider a system of $n$ ordinary differential equations defined as:

$\frac{d \mathbf{x}(t)}{d t}=\mathbf{F}(\mathbf{x}(t))$

where $\mathbf{x}(\mathrm{t})=\left[x_{1}(t), x_{2}(t), \ldots, x_{n}(t)\right]$ in $R^{n}$ and $\mathbf{F}=\left[F_{1}, \ldots, F_{n}\right]$ is a smooth nonlinear function of $\mathbf{x}$, i.e. the existence and uniqueness properties hold. At time $t>0$ the initial condition $\boldsymbol{x}(0)$ finds itself at some new point $\boldsymbol{x}(t)$. Similarly, all initial conditions lying in a certain region $\Gamma(0)$ find themselves in another region $\Gamma(t)$ after time $t$. If we let $V(t)$ denote the volume of the region $\Gamma(t)$, then a strong version of Liouville's theorem [15] states that: 
$\frac{d V(t)}{d t}=\int_{\Gamma(t)} \operatorname{tr}[J(x)] d x_{1} \ldots d x_{n}$

where

$\operatorname{tr}[J(x)]=\operatorname{div}[J(x)]=\frac{\partial F_{1}(x)}{\partial x_{1}}+\ldots+\frac{\partial F_{n}(x)}{\partial x_{n}}$

Assuming that our n-dimensional volume is small enough that the divergence of the vector field is constant over $V(t)$, then

$\frac{d V(t)}{d t}=V(t) \cdot \operatorname{div}[J(x)]$

and hence,

$\int_{0}^{t} \frac{d V(\tau)}{V(\tau)}=\int_{0}^{t} \operatorname{div}[J(x)] d \tau$

which means that the initial phase space volume $V(0)$ shrinks (grows) with time in $R^{n}$ as:

$V(t)=V(0) \cdot \exp \left[\int_{0}^{t} \operatorname{div}[J(x)] d \tau\right]$

Hence, for the case of a system given by Eq. (1), the rate of change of an infinitesimal volume $V(t)$ following an orbit $\boldsymbol{x}(t)$ is given by the divergence of the flow which is locally equivalent to the trace of the Jacobian. If $\operatorname{div}[\mathbf{F}(\mathbf{x})]<0$ then the flow of trajectories is volume-contracting, if $\operatorname{div}[\mathbf{F}(\mathbf{x})]>$ 0 the flow is volume-expanding, and if $\operatorname{div}[\mathbf{F}(\mathbf{x})]=0$ then the flow is volume-preserving. From Eq. (6), it is possible to write [5]:

$\operatorname{div}[J(x)]=\frac{\dot{V}(t)}{V(t)}$

\section{Batch Reactors}

The mass and energy dimensionless balances $\left(z, \theta, \theta_{w}\right)$ that describe the dynamic behaviour of a well-stirred controlled batch reactor (BR) in which the reaction: $A \rightarrow B$ occurs can be written as [7]:

$\frac{d z}{d \tau}=D a \cdot \exp \left(\frac{\theta \cdot \gamma}{\gamma+\theta}\right)(1-z)^{n}$

$\frac{d \theta}{d \tau}=B \cdot D a \cdot \exp \left(\frac{\theta \cdot \gamma}{\gamma+\theta}\right)(1-z)^{n}-S t\left(\theta-\theta_{w}\right)$

$\frac{d \theta_{w}}{d \tau}=\delta \cdot S t\left(\theta-\theta_{w}\right)+Q_{c}$

and the initial conditions (ICs):

$z=0, \theta=0$ and $\theta_{w}=0$ at $\tau=0$

where we have introduced the following variables:

$z \frac{C^{i}-C}{C^{i}} ; \theta=\frac{T-T^{i}}{T^{i}} \cdot \gamma ; \theta_{w}=\frac{T_{w}-T^{i}}{T^{i}} \cdot \gamma ; \tau=t$

and dimensionless parameters:

$\gamma=\frac{E}{R \cdot T^{i}} ; \quad D a=k\left(T^{i}\right) \cdot\left(C^{i}\right)^{n-1} ; \quad B=\frac{(-\Delta H) \cdot C^{i}}{\rho \cdot V \cdot C p \cdot T^{i}} \cdot \gamma ;$

$S t=\frac{U \cdot S}{\rho \cdot V \cdot C p} ; \delta=\frac{\rho \cdot V \cdot C p}{\rho_{w} \cdot V_{w} \cdot C p_{w}} ; Q_{c}=\frac{q_{c}}{\rho_{w} \cdot V_{w} \cdot C p_{w} \cdot T^{i}} \cdot \gamma$
By using these definitions, the system behaviour is characterized by six parameters: $n, \gamma, D a, B, S t, \delta$ and $Q_{c}$ (see Notation). The first three depend on the kinetics. $B$ is the dimensionless heat of reaction, $S t$ is the Stanton number and $Q_{c}$ is the dimensionless heating/cooling power, which is the control variable. In this work, and in order to compare with a traditional control scheme, we have used a two proportional controller loops scheme where the control of reactor temperature is accomplished by the master controller, whereas the reactor jacket temperature is controlled using a slave controller. This configuration is typical of reaction calorimeters [16] and it has been used in pilot plant reactors [17]. In this case, it is possible to write [7]:

$Q_{c}=K p_{2}\left(\theta_{w}^{s p}-\theta_{w}\right)$

where the jacket temperature set point is given by:

$\theta_{w}^{s p}=\theta^{s p}+K p_{1}\left(\theta^{s p}-\theta\right)$

depending of the predefined reactor temperature set-point, $\theta^{s p}$. In order to consider control saturation during transients, the manipulated variable, $Q_{c}$, is constrained as follows: if the required $Q_{c}$ is higher than the maximum heating capacity of the system, this parameter takes this maximum value, $Q_{c}=Q_{\text {cmax }}$; and if $Q_{c}$ is lower than the maximum cooling capacity then the parameter takes this minimum value, $Q_{c}=Q_{\text {cmin }}$.

The analytical divergence of this system of equations can be obtained by calculating the trace of the Jacobian matrix, i.e. $\operatorname{div}\left(z, \theta, \theta_{w}\right)=j_{11}+j_{22}+j_{33}$.

$j_{11}=-D a \cdot \exp \left(\frac{\theta \cdot \gamma}{\gamma+\theta}\right) \cdot n \cdot(1-z)^{n-1}$

$j_{22}=B \cdot D a \cdot \exp \left(\frac{\theta \cdot \gamma}{\gamma+\theta}\right) \cdot \frac{\gamma^{2}}{(\gamma+\theta)^{2}}-S t$

$j_{33}=-\delta \cdot S t-K p_{2}$

When the controller works in saturation the term $j_{33}$ is modified as follows:

$j_{33}=-\delta \cdot S t$

\section{State Space Divergence Reconstruction Using Experi- mental Data}

In order to calculate on-line, the divergence without the need to know the differential equations of the system we have used the theory of embedding. The theory of embedding is a way to move from a temporal time series of measurements, $s(t)=h[\boldsymbol{x}(t)]$, which is related to the state variables, $\boldsymbol{x}(t)$, by a unknown function, $h$, to a state space similar -in a topological sense- to that of the underlying dynamical system we are interested in analysing. Techniques of state space reconstruction were introduced by [18-19], who showed it is possible to address this problem using time delay embedding vectors of the original measurements, i.e. $\{s(t), s(t-\Delta t), s(t-$ $\left.2 \Delta t), \ldots, s\left(t-\left(d_{E^{-}}-1\right) \Delta t\right)\right\}$.

The extension of this theory for the case of batch and semibatch chemical reactors is complicated by the dynamic nature (nonstationarity) of the system that implies embedding parameters, i.e. time delay, $\Delta t$ (the lag between data 
when reconstructing the state space), and embedding dimension, $d_{E}$ (the dimension of the space required to unfold the dynamics) are changing during the process. However, as it was discussed in [20] and [9] that an appropriate value for these parameters can be chosen to allow state space reconstruction. Furthermore, several methods of reconstruction of state space: time delay embedding vectors; derivative coordinates and integral coordinates may be used [9]. However, the more robust to noise is the one based on delayed vectors of the reactor temperature, i.e. $\{\theta(t), \theta(t-\Delta t)\}$ [12] or the difference between reactor and jacket temperatures, i.e. $\{\theta(t)$ $\left.\theta_{w}(t), \theta(t-\Delta t)-\theta_{w}(t-\Delta t)\right\}[10]$, and for the cases studied an embedding dimension of two is sufficient to reconstruct the divergence as it was shown in [12], $d_{E}=2$.

Numerically, $V(t)$ may be calculated, assuming that the time step from one point to another in the time series is short enough that the Jacobian of the system has not substantially changed, using the determinant between close points in state space as [12]:

$V(t)=\mid \operatorname{det}\left[\begin{array}{cc}\theta(t)-\theta(t-\Delta t) & 0 \\ 0 & \theta(t-\Delta t)-\theta(t-2 \Delta t)\end{array}\right]$

The comparison between experimental analytical divergence for a bench-scale pilot plant shows that it is possible to reconstruct the divergence [12]. However, "safe" experiments having low values of $V(t)$ and small variations of $\Delta V(t)$ are more affected by noise. In dissipative systems the
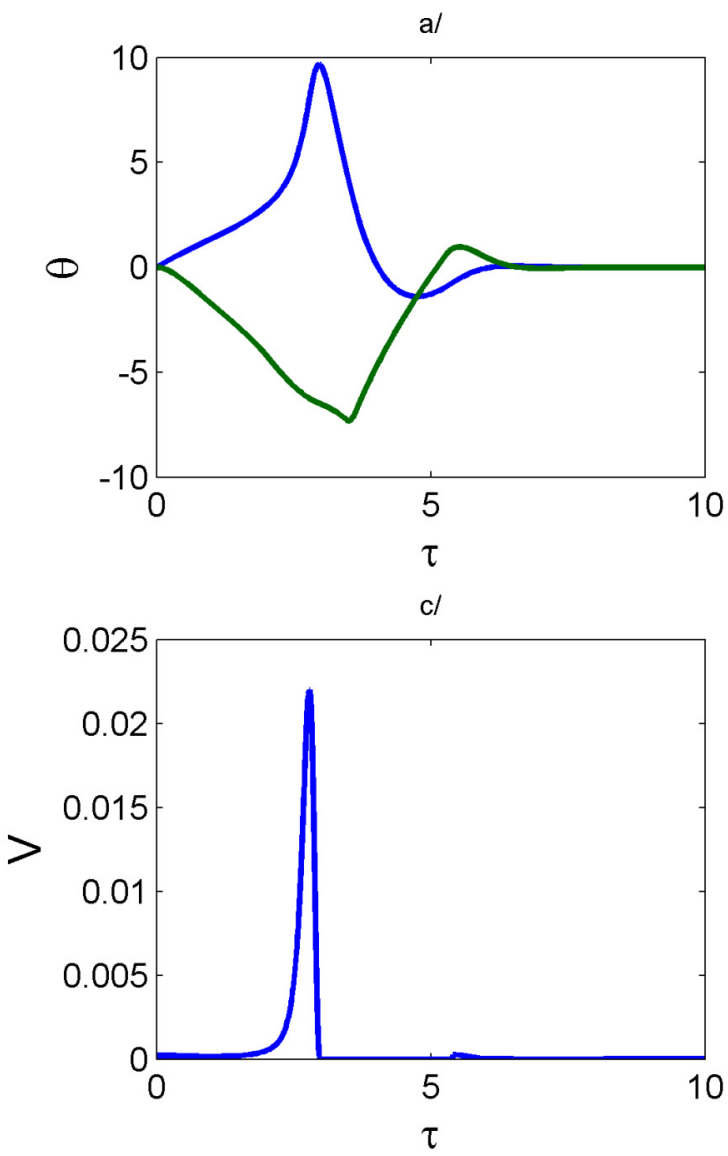

state space volume contracts and therefore $V(t)$ and $\dot{V}(t)$ rapidly tend to become zero and produce artefacts when introduced as denominator in Eq. (7), for this reason, normally we will use $V(t)$ and $\Delta V(t)=V(t)-V(t-\Delta t)$ separately, avoiding the need to divide the two small numbers. In addition there is a limit for the calculation of $V(t)$ in order to avoid numerical problems when the temperatures do not change appreciably. In this case the limit value for $\mathrm{d} \theta / \mathrm{dt}$ is 0.01 .

\section{RESULTS AND DISCUSSION}

The typical behaviours of the controlled BR are shown in Figs. $(\mathbf{1 , 2})$ for the case of runaway conditions and in Figs. $(3,4)$ for the simulation in non-runaway conditions which, in this case, depend on the thermal reaction number $B$.

The simulation parameters in Figs. $(\mathbf{1}, \mathbf{4})$ were $D a=0.05$; $\gamma=10 ; n=1 ; S t=1 ; \delta=0.24 ; K p_{1}=2 ; K p_{2}=3 ; Q_{\text {cmax }}=4.0$; $Q_{c m i n}=-5.0$. The only difference is the thermal reaction number that in the first case, Figs. $(\mathbf{1 , 2})$, is $B=29$, whereas in the non-runaway case, Figs. $(\mathbf{3 , 4})$, is $B=20$. As can be observed, in the first case, the system is not able to control the rate of heat production and the system reach saturation (Fig. 2), whereas in the second case the cooling system has enough power capacity to control the rate of heat release (Fig. 4) and the system does not reach saturation.

The reconstructed state space volume, $V$, Eq. (17), and $\Delta V$ - using and embedding dimension of two, a time delay $\Delta t$ $=10^{-2}$, and the algorithm developed in [12] - are shown for both situations in Figs. (1c,d and 2 c,d). As it can be ob-
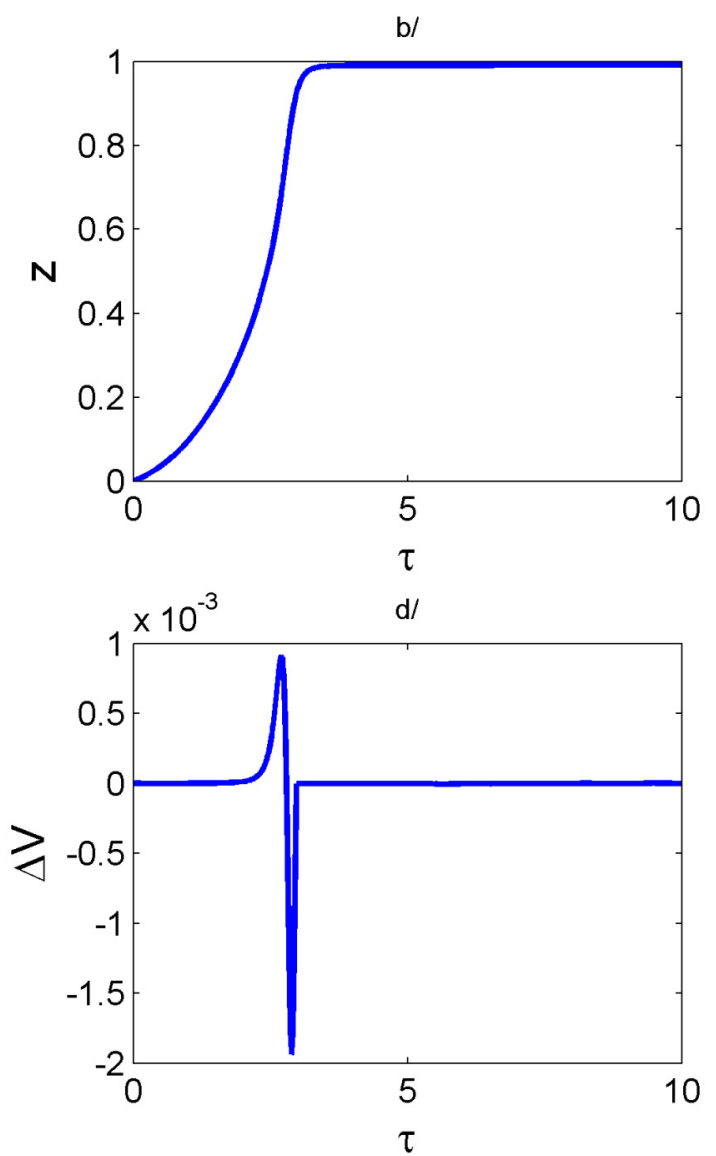

Fig. (1). Runaway simulation. a/Reactor and jacket temperatures; b/conversion; c/Reconstructed state space volume; d/Reconstructed state space volume change. 
served that there are several orders of magnitude between $\Delta V$ for the runaway and non-runaway situation and, therefore, it is possible to define a limit for distinguishing between both cases.

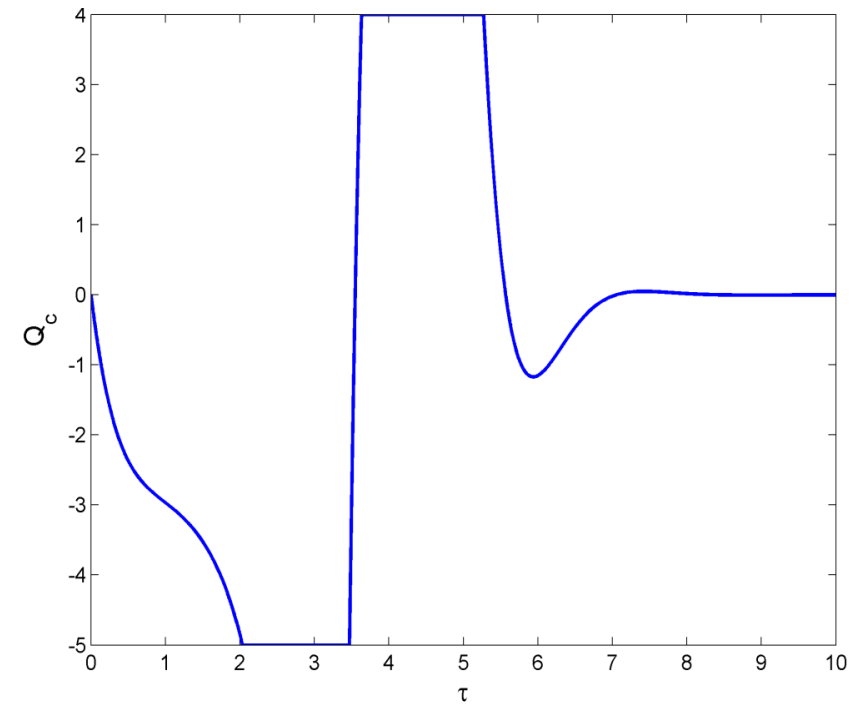

Fig. (2). Runaway simulation: cooling/heating power provided by the control system.

\section{The $\Delta V$ as an On/Off Controller}

Let us first study the case in which $\Delta V$, which was the criterion used by [12] to detect runaway initiation, is used as an active system when a runaway initiation is detected. In this case, when $\Delta \mathrm{V}>\lim _{\text {runway }}$, the control system adds an additional term to Eq. (11) in the form of:

$$
Q_{c}=K p_{2}\left(\theta_{w}^{s p}-\theta_{w}\right)+K_{\Delta V} \cdot(0-\Delta V)
$$

In this case the limit value for $\Delta V$ was established as $1.010^{-6}$. Fig. (5) shows the dynamic behaviour of reactor and jacket temperatures as well as conversion and state space reconstructed volume and its difference for the case of using $\Delta V$ as an on/off controller. As can be seen in this case the maximum dimensionless reactor temperature drops from 9.7 (Fig. 1a) to 4.1. In addition, as can be observed in Fig. (6) the behaviour of the cooling system is quite similar. The observed differences in temperatures are due to the high parametric sensitivity in runaway conditions, and therefore, under such conditions a small change would suffice to change the dynamic behaviour of the system.

\section{The Time-Step Volume Preserving Control Approach}

When operating chemical reactors, the main objective is to maximise production and in parallel to increase the safety. In this sense, if we are in a dangerous situation when div $>0$ [5] and we are in a safe situation when $d i v<0$, it seems natural to conclude that if we want to maximise production (reducing time between batches) and work on safety conditions we should impose $d i v=0$ as our controller set-point. However, for dissipative systems like BR, the state space volume
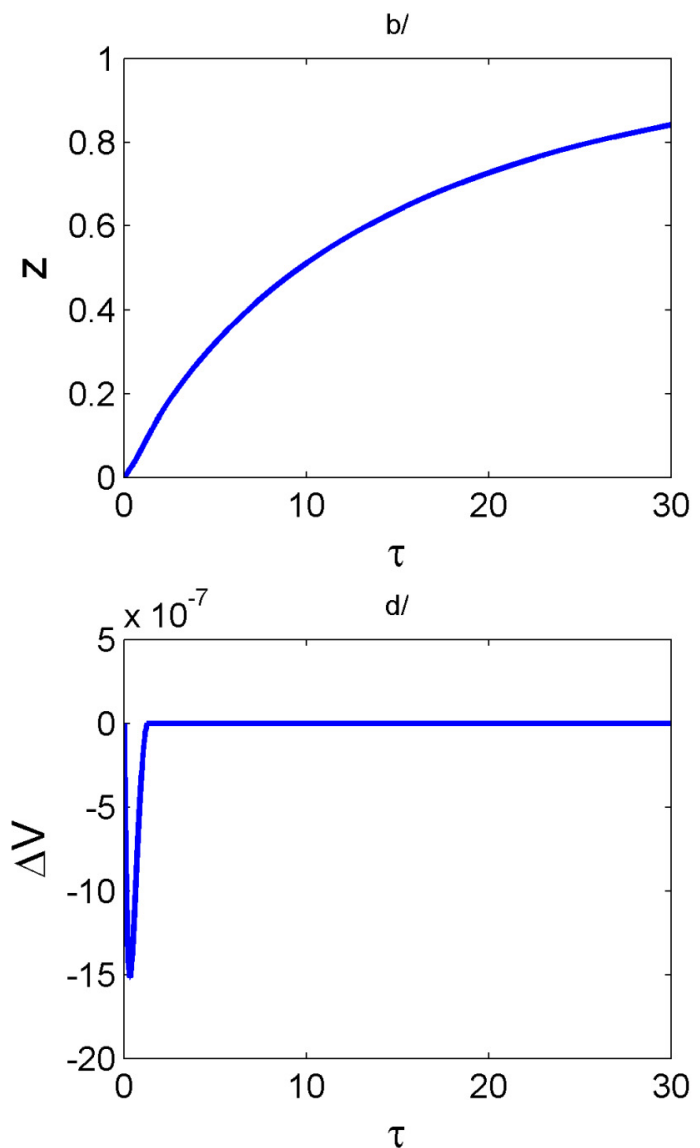

Fig. (3). Non runaway simulation. a/ Reactor and jacket temperatures; b/ conversion; c/ Reconstructed state space; d/ Reconstructed state space volume change. 


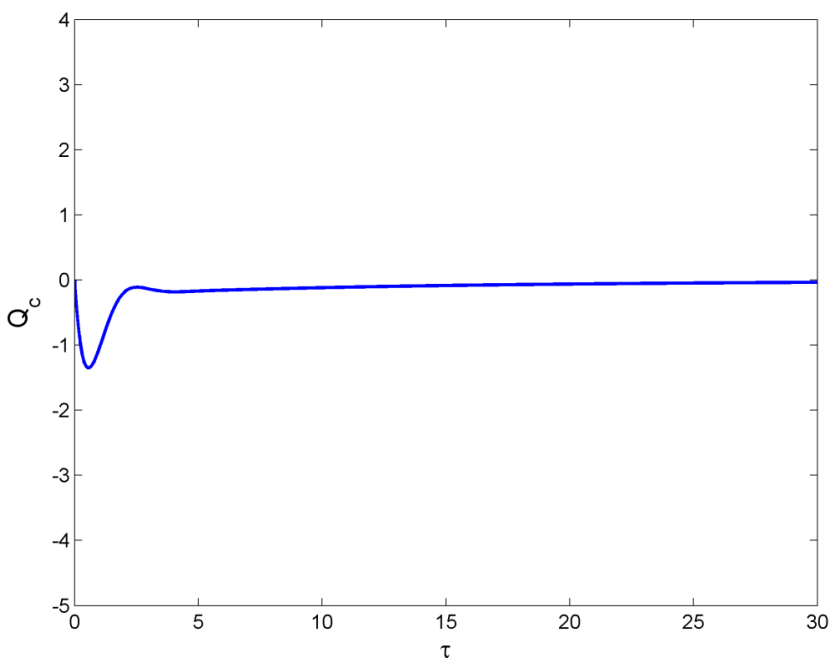

Fig. (4). Non-runaway simulation: cooling power provided by the control system.

tends to contract and it becomes too small for its practical use in calculating the divergence, this normally happens towards the end of the reaction. Fig. (7) shows the analytical divergence for the runaway and non-runaway reactions. As can be seen, when the reaction has been completed, the divergence of the BR becomes negative, so the system will always try to increase the reactor temperature if we set div $=$ 0 as the set-point [14]. To avoid this problem, we have used a mixed control system that tries to keep $V(t)$ and $\Delta V(t)$ close to zero, when it is possible to calculate them (see Methods and Approach Section) and applies the traditional control scheme when $V(t)$ or $\Delta V(t)$ cannot be calculated due to their small values and the associated numerical problems. Therefore the control strategy is:

$Q_{c}=K_{p V} \cdot(0-V(t))+K_{d V}(0-\Delta V(t))$

If the state space volume or its variation cannot be calculated then we apply Eqs. (11)-(12).

Figs. $(\mathbf{8 , 9})$ show the result of this controller applied to the runaway process previously described in Fig. (1), whereas Figs. $(\mathbf{1 0 , 1 1 )}$ refer to the non-runaway process described in Fig. (3). In this case, to reconstruct the state space volume, we have used the temperature difference between the reactor and the jacket, i.e. $\left\{\theta(t)-\theta_{w}(t), \theta(t-\Delta t)-\theta_{w}(t-\Delta t)\right\}$. As it can be seen, the fast process is slowed down by an effective control system, whereas the slow process is accelerated and both, temperature profiles and conversion, become quite similar even though the dimensionless heat of reaction is quite different.

The dynamics of the heating/cooling power is depicted in Figs. $(9,11)$. The reconstruction using reactor and jacket temperature seems more appropriate for controlled reactors than using only reactor temperature since both are state variables [10]. However, it may be not possible to use it when the heating-cooling fluid is used in other applications as in a large number of industrial installations [13] and therefore, the dynamics of the jacket temperature does not reflect perfectly the dynamics of the controlled reactor.
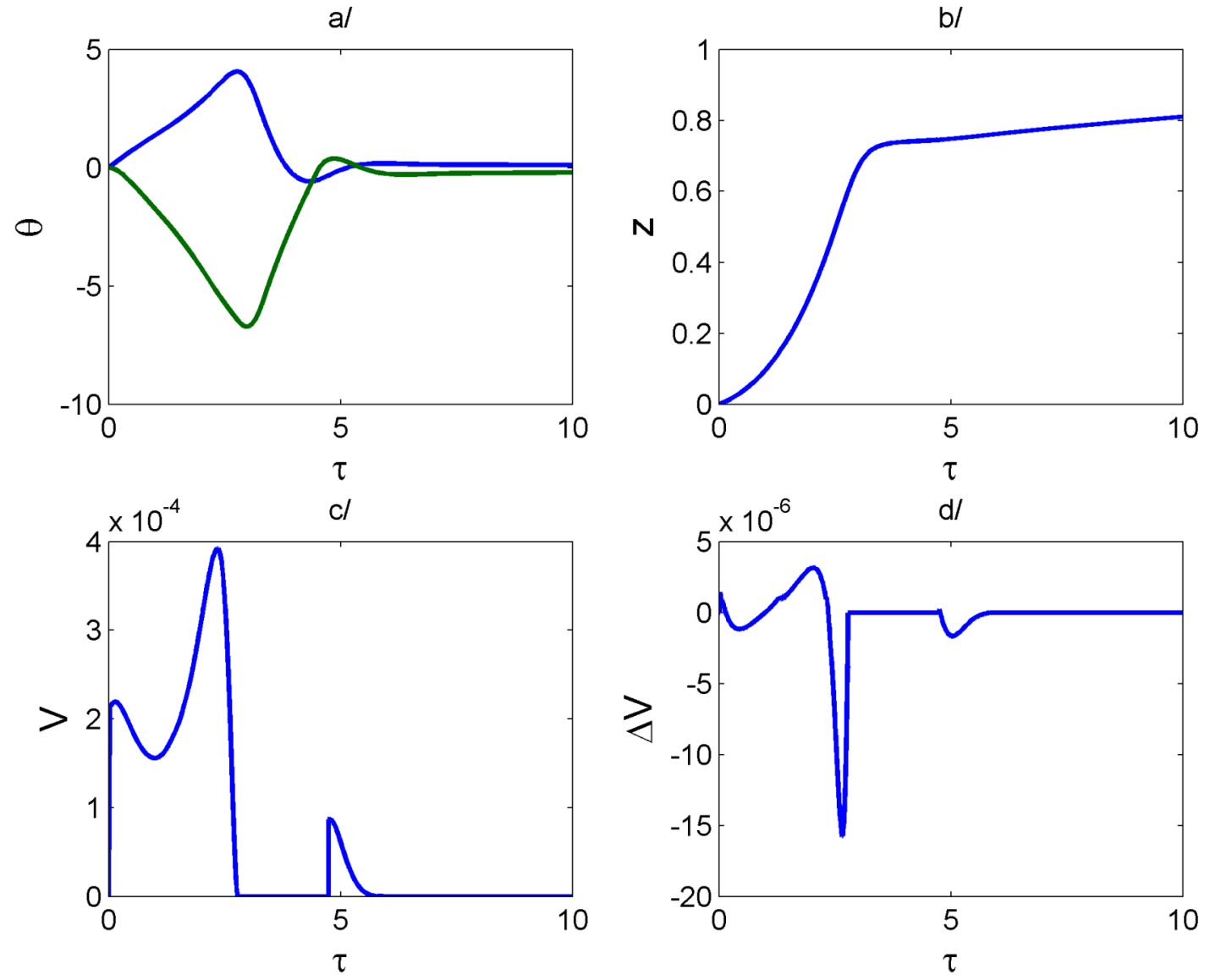

Fig. (5). Simulation for the runaway case, see Fig. (1) with $\Delta V$ as an on/off controller. a/ Reactor and jacket temperatures; b/conversion; c/Reconstructed state space; d/Reconstructed state space volume change. Same parameters as Fig. (1). 


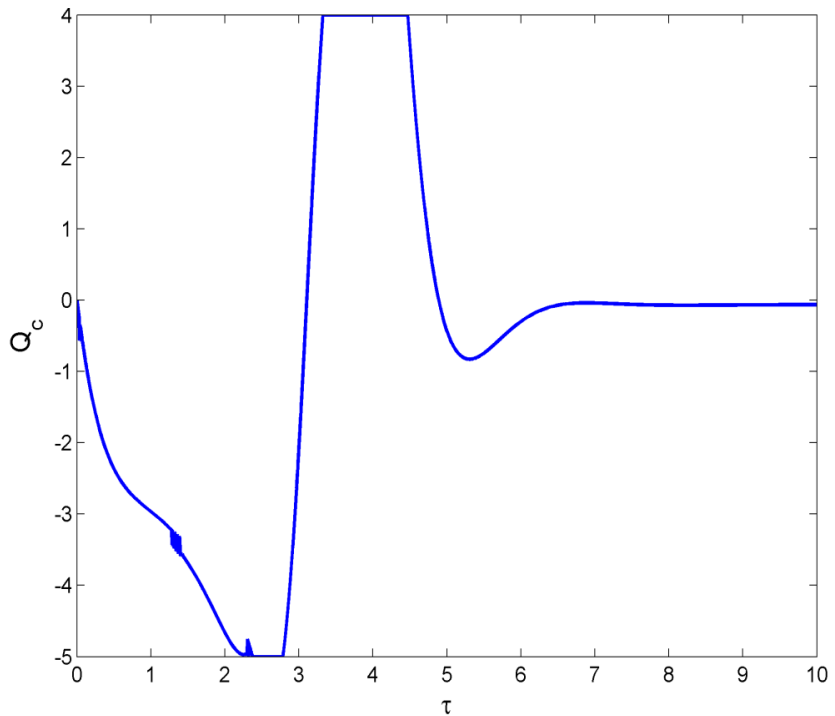

Fig. (6). Cooling power provided by the control system using $\Delta V$ as an on/off controller.

a/
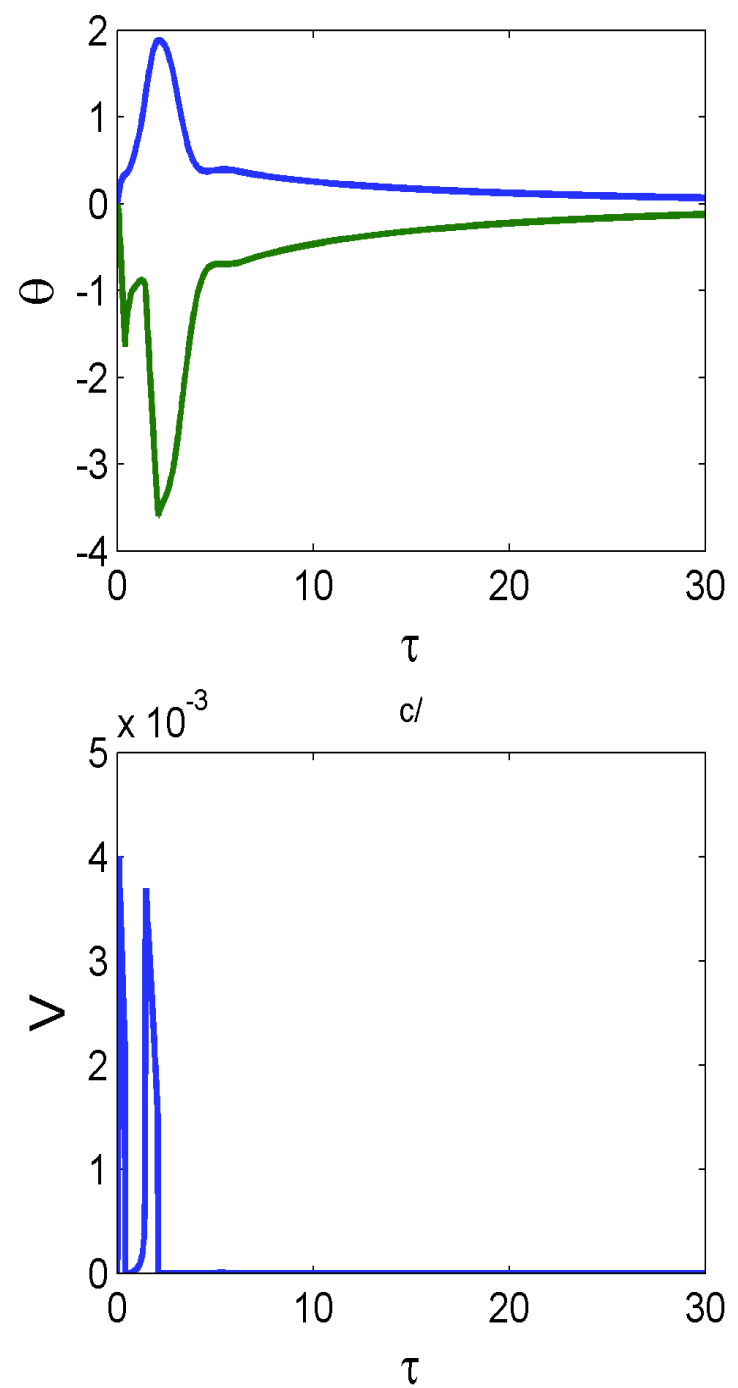
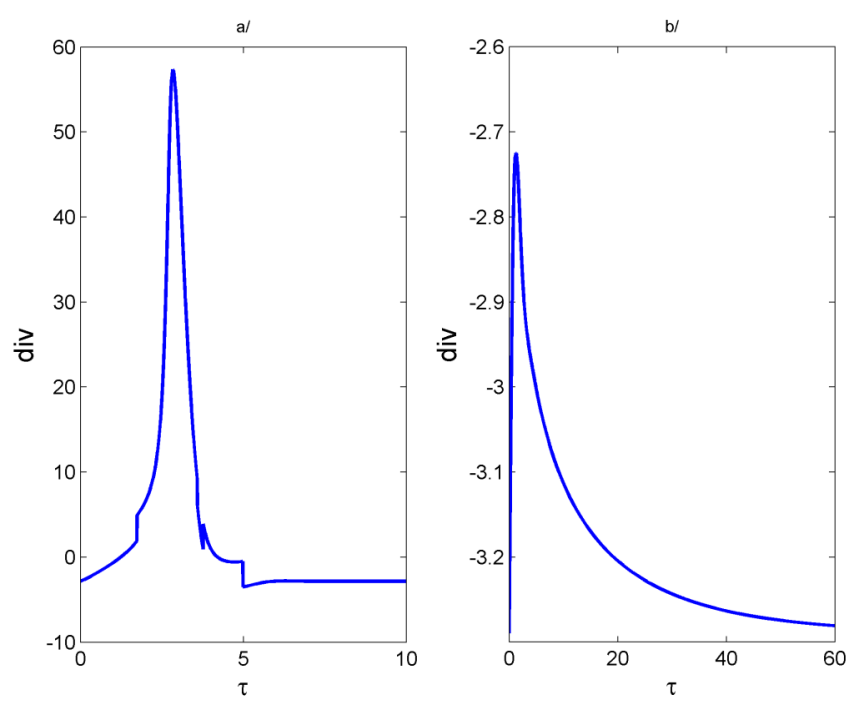

Fig. (7). Analytical divergences for: a/ runaway (Fig. 1); b/ nonrunaway experiments (Fig. 3).
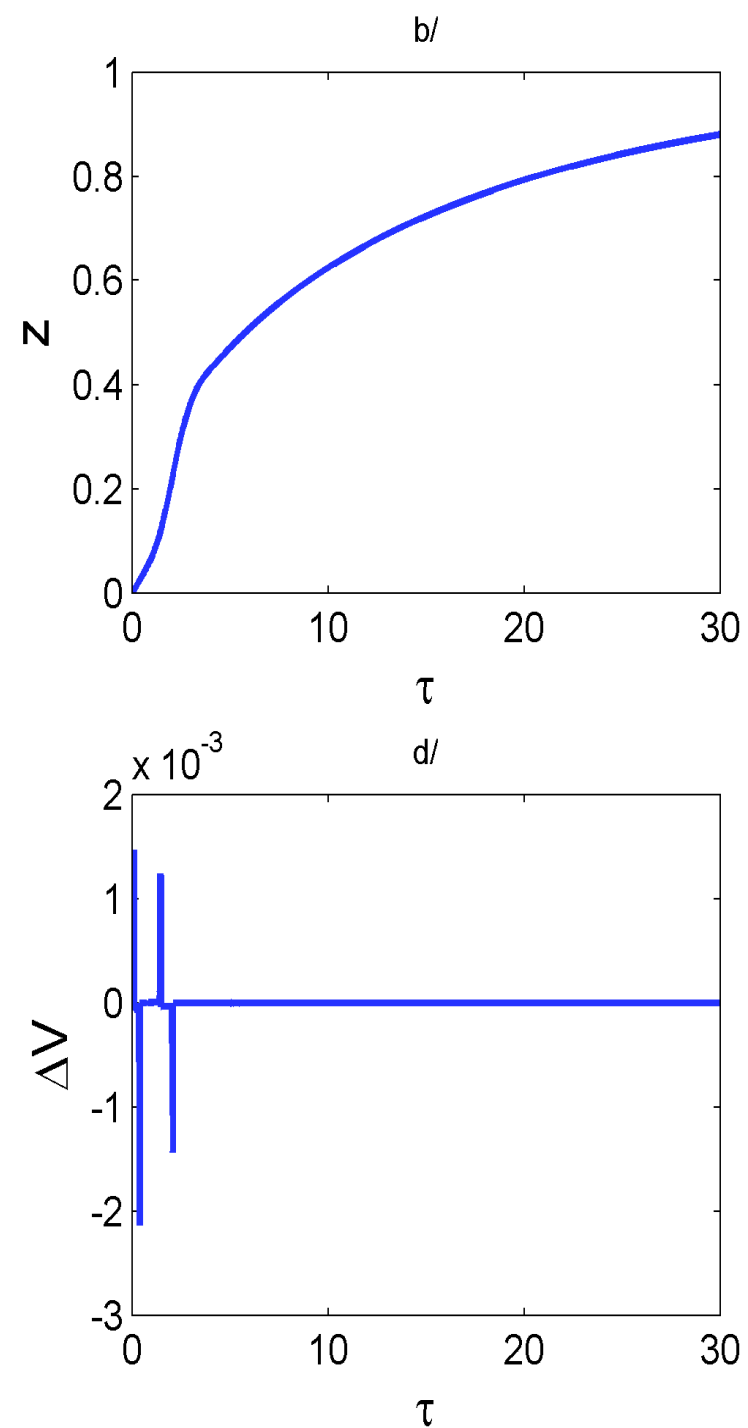

Fig. (8). Simulation for the runaway situation, see Fig. (1), with Eq. (19) as controller. a/ Reactor and jacket temperatures; b/conversion; c/ Reconstructed state space; $d$ / Reconstructed state space volume change. 


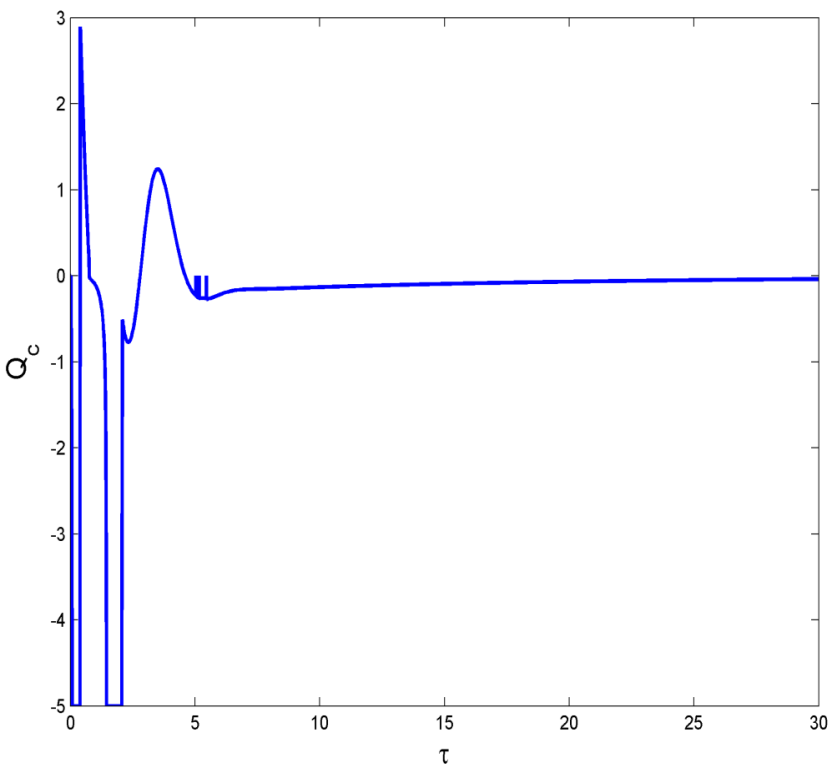

Fig. (9). Cooling power provided in Fig. (8) by the control system using Eq. (19) as controller.

al
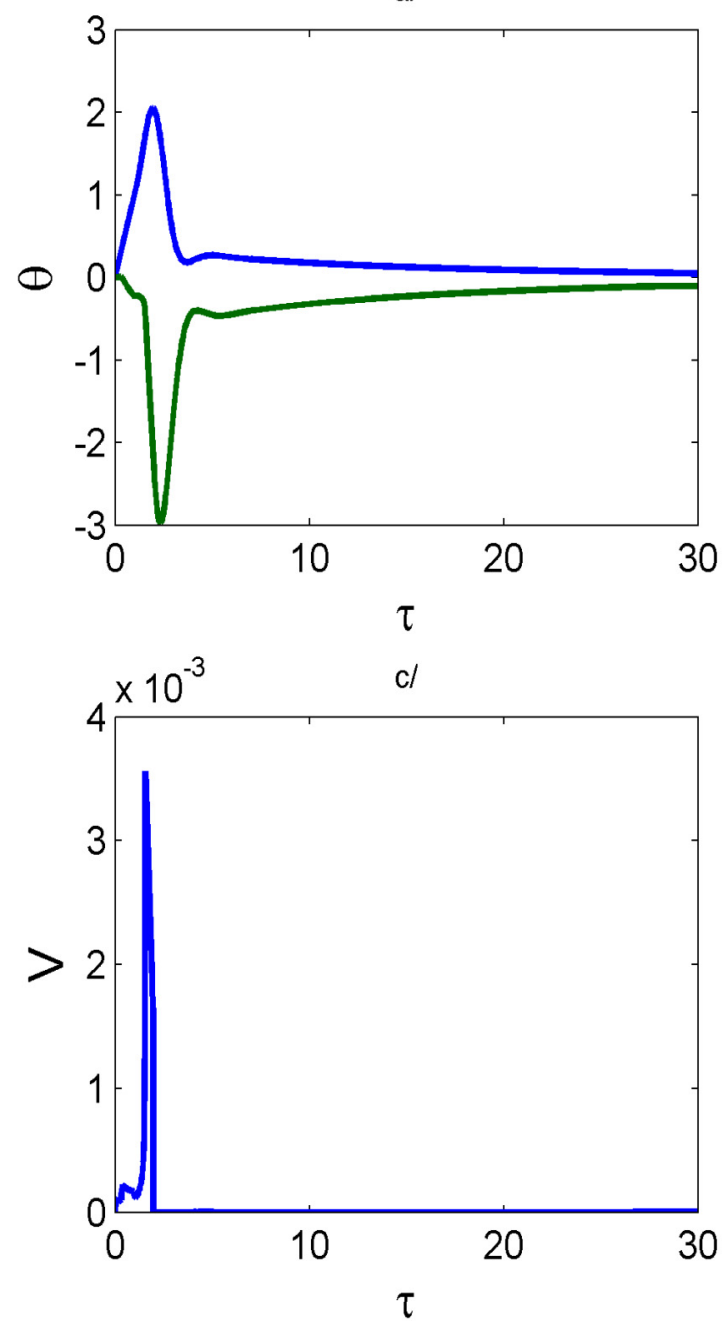

As an example, Figs. $(\mathbf{1 2 , 1 3 )}$ show the results obtained applying Eq. (19) to $V$ and $\Delta V$ reconstructed using $\{\theta(t), \theta(t-$ $\Delta t)\}$. Like in the other case it is always possible to define a control strategy that will optimize safety and performance.

\section{CONCLUSIONS}

We present a general control and optimization strategy based on time-step state space volume preservation. This is performed by maintaining the divergence of the system close to zero. The on-line implementation, using simulation results, is shown for batch chemical reactors. The divergence of these systems is reconstructed on-line using reactor and jacket temperature measurements only and applying state space reconstruction techniques. The simulated results are compared with a traditional control scheme. This kind of control allows to operate in safety conditions and to optimize the performance of the process by accelerating the slow reactions and reducing batch times. However, as dissipative systems have an exponential state space volume contraction, and the divergence of these systems will become negative as $t \rightarrow \infty$, it is necessary to combine it with the traditional scheme to avoid unnecessary heating at the end of the batch process when the reaction get finished.

b/
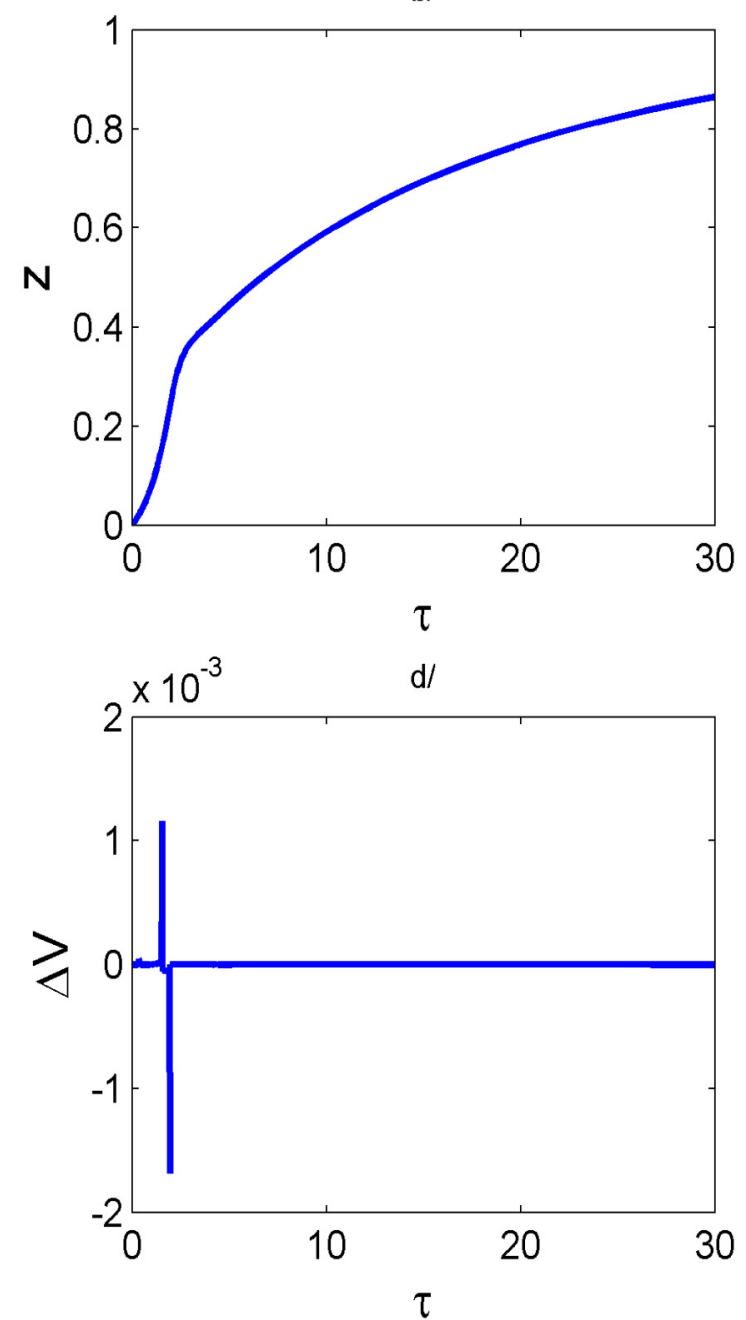

Fig. (10). Simulation for the nonrunaway situation, see Fig. (3), with Eq. (19) as controller. a/ Reactor and jacket temperatures; b/ conversion; c/ Reconstructed state space; $\mathrm{d}$ / Reconstructed state space volume change. 


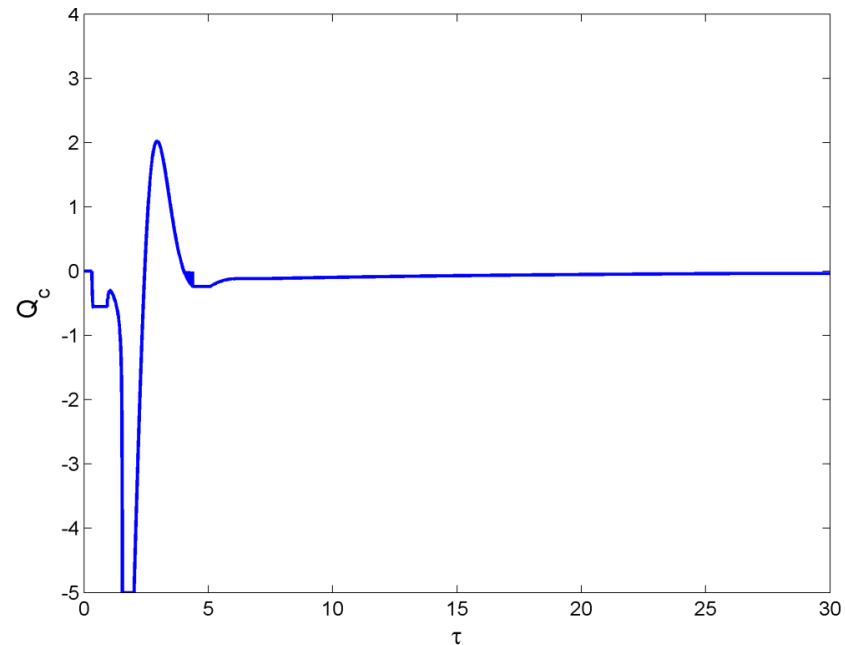

Fig. (11). Cooling power provided in Fig. (10) by the control system using Eq. (19) as controller.
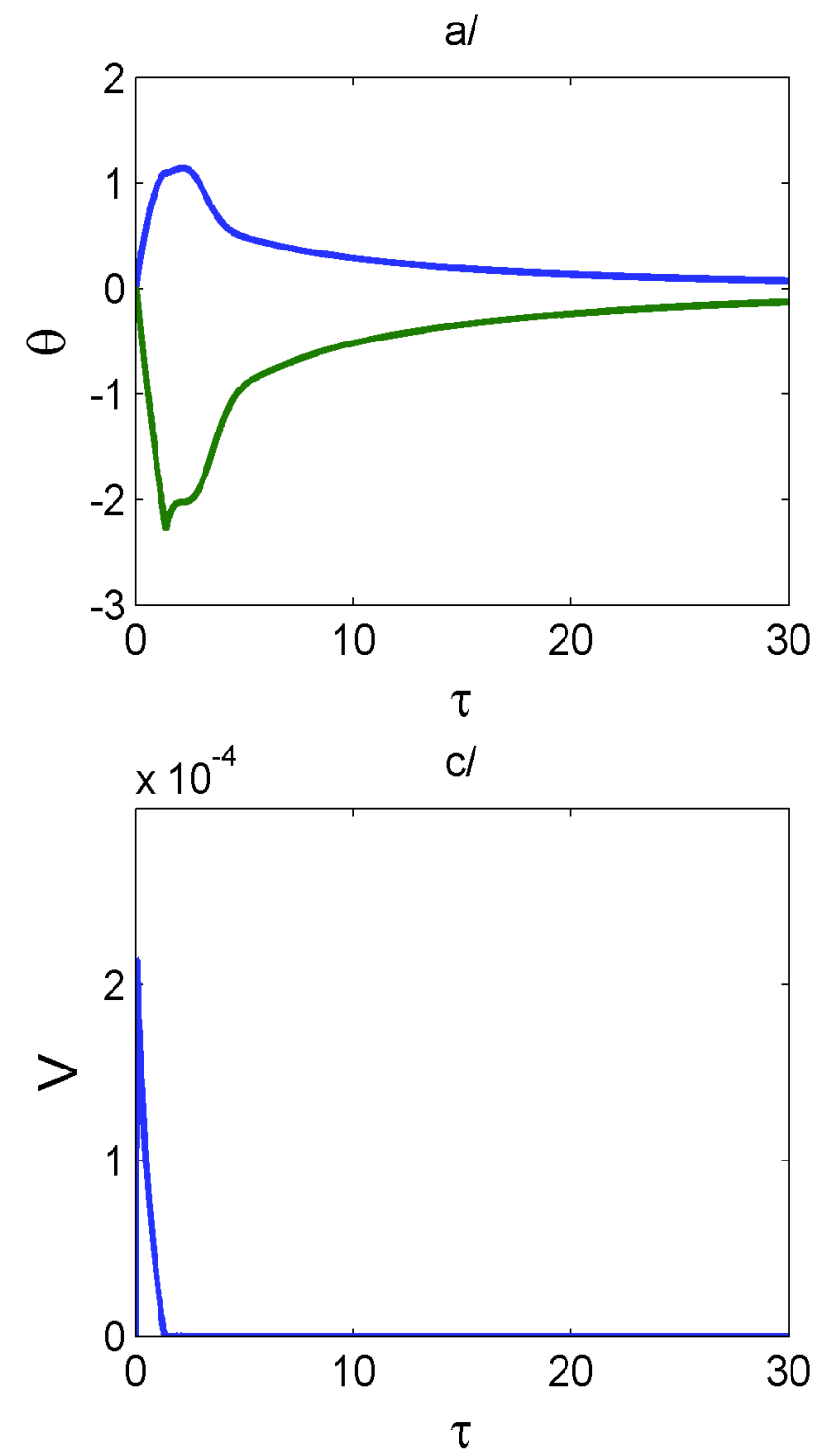

The next step of this research will be the real implementation of these control strategies first on a bench scale, then on a pilot plant and then on an industrial reactor. An important aspect to be tested when using this approach is its robustness against noise and operator manipulations in real plants. Noise is always a problem in any control strategy. In this case since our aim is to reconstruct the divergence of the $\mathrm{BR}$, non-linear noise reduction schemes [21-22] have proven to outperform traditional filtering techniques [9]. In addition, the approach is easily applicable to other types of reactors and it is independent of the chemical process carried out in the reactor.

Since the proposed control scheme, based on trying to convert a dissipative system into a conservative system at each time step, is quite general then, probably, it can be of interest in other fields than chemical engineering.
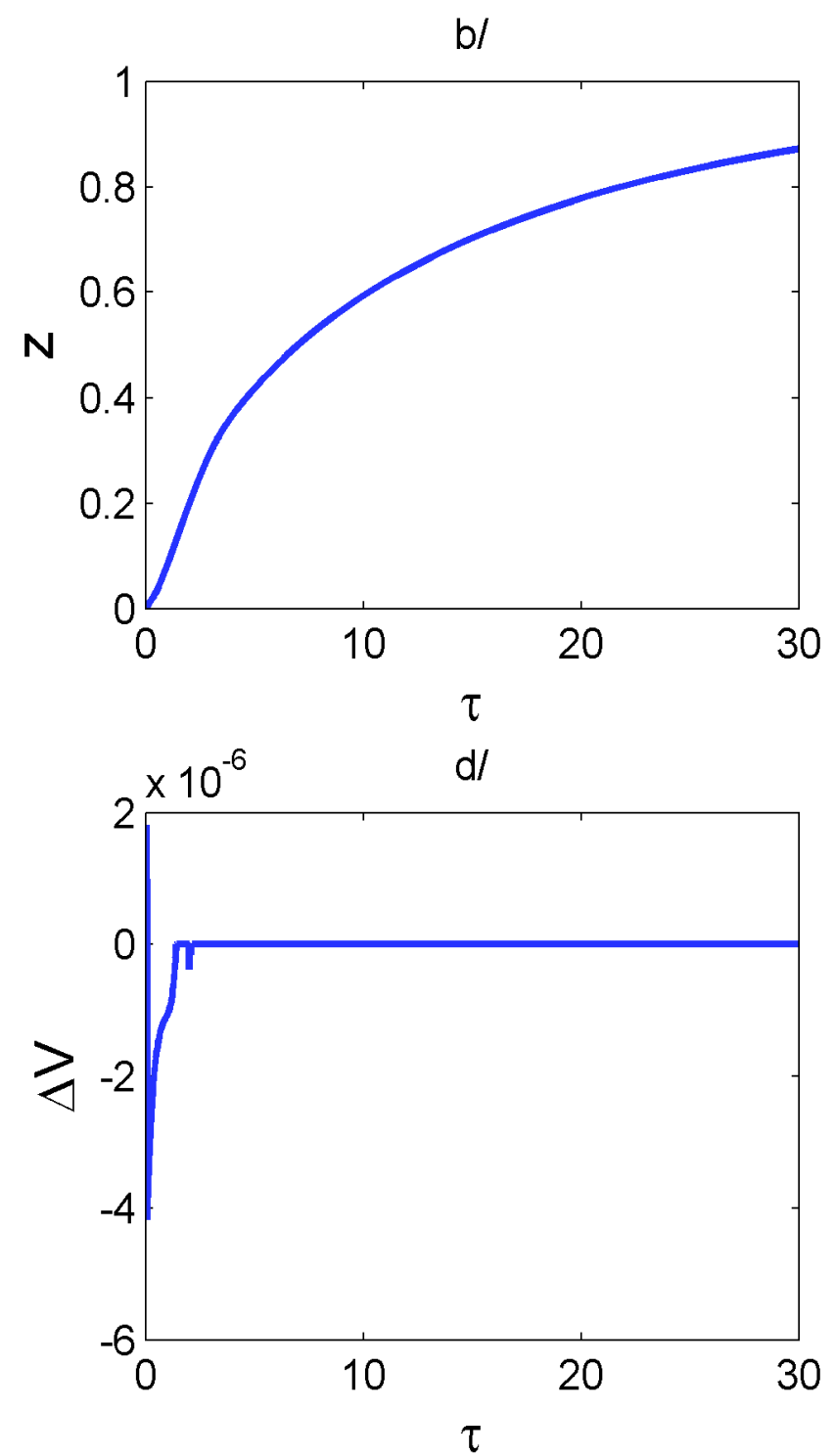

Fig. (12). Simulation for the runaway situation, see Fig. (1), with Eq. (19) as controller using only reactor temperature for state space reconstruction. a/ Reactor and jacket temperatures; b/conversion; $c$ / Reconstructed state space; $d$ / Reconstructed state space volume change. 


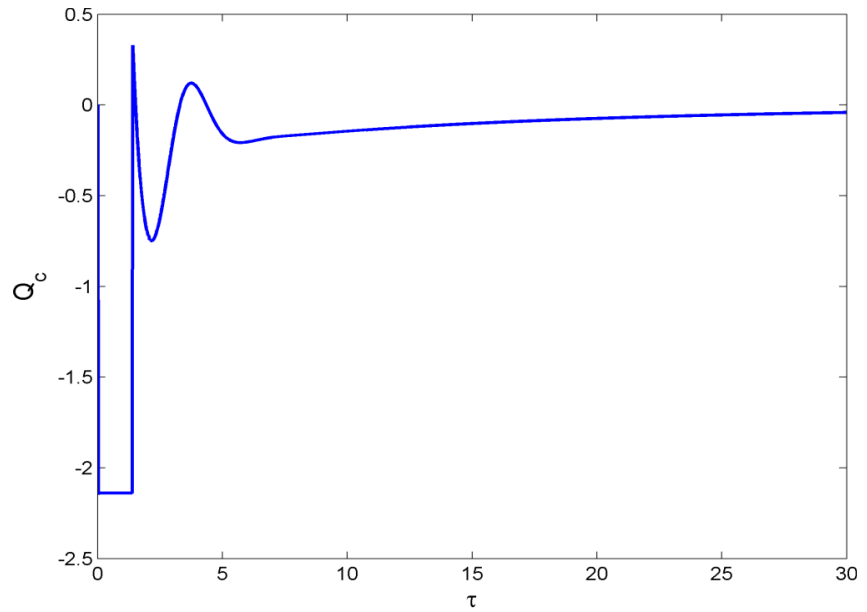

Fig. (13). Cooling power provided in Fig. (12) by the control system using Eq. (19) as controller.

\section{NOTATIONS}

$B$ Dimensionless heat of reaction parameter

$C p \quad$ Mean specific heat of reaction mixture $\mathrm{kJ}^{-1} \cdot \mathrm{kg}^{-1}$

C Concentration of reactant, $\mathrm{kmol}^{\prime} \mathrm{m}^{-3}$

Da Damköhler number

E Activation energy, $\mathrm{kJ}^{\prime} \mathrm{mol}^{-1}$

$k \quad$ Reaction rate constant, $\left(\mathrm{kmol}^{-3} \mathrm{~m}^{-3}\right)^{(1-\mathrm{n})} \mathrm{s}^{-1}$

$n \quad$ reaction order

$q \quad$ Heating/cooling power, $\mathrm{kJ} \mathrm{s}^{-1}$

$Q \quad$ Dimensionless heating/cooling power

$R \quad$ Universal gas constant

St Stanton number

$S \quad$ Heat exchange surface area, $\mathrm{m}^{2}$

$t$ Time, s

$T \quad$ Temperature, $\mathrm{K}$

$U \quad$ Heat transfer coefficient, $\mathrm{kJ} \cdot\left(\mathrm{m}^{2} \cdot \mathrm{K} \cdot \mathrm{s}\right)^{-1}$

$V \quad$ Reactor volume or state space volume

$z \quad$ Conversion

\section{Greek Symbols}

$\delta \quad$ Cooling dimensionless time,

$\Delta H \quad$ Heat of the reaction, $\mathrm{kJ}^{\circ} \mathrm{kmol}^{-1}$

$\rho \quad$ Density of the mixture

$\gamma \quad$ Dimensionless activation energy

$\theta$ dimensionless temperature

$\tau \quad$ dimensionless time

\section{Superscripts}

$i \quad$ initial condition

sp set-point

\section{Subscripts}

j,w jacket

$\mathrm{r}$ reactor

\section{REFERENCES}

[1] P. Hugo, "Start-Up and Operation of Exothermic Batch Processes", Chem. Ing. Technol., vol. 52, pp. 712-722, 1980.

[2] J. Steinbach, 1989. "Fundamentals/Theory of Runaway Chemical Reactions", in Proceedings of the Conference on Techniques for Assessment of Chemical Reaction Hazards, 1989, pp. 40-63.

[3] M. Steensma, and K.R. Westerterp, "Thermally safe operation of a semi-batch reactor for liquid-liquid reactions", Ind. Eng. Chem. Res. vol. 29, pp. 1259-1270, 1990.

[4] M. Steensma, and K.R. Westerterp, "Thermally safe operation of a semi-batch reactor for liquid-liquid reactions: fast reactions", Chem. Eng. Technol., vol.14, pp. 367-375, 1991.

[5] F. Strozzi, J. M. Zaldívar, A. Kronberg, and K. R. Westerterp, "Online runaway prevention in chemical reactors using chaos theory techniques", AIChE J., vol. 45, pp. 2394-2408, 1999.

[6] J. M. Zaldívar,. and F. Strozzi, "On-line early warning detection system of runaway initiation using chaos theory techniques" EU Patent 0882499,2003

[7] J. M. Zaldívar, J. Cano, M. A. Alós, J, Sempere, R. Nomen, D. Lister, G. Maschio, T. Obertopp, E. D. Gilles, J. Bosch, and F. Strozzi, "A general criterion to define runaway limits in chemical reactors", $J$. Loss Prev. Process Ind., vol. 16, pp.187-200, 2003.

[8] J. Bosch, F. Strozzi, D. Lister, G. Maschio, and J.M. Zaldívar, "Sensitivity Analysis in Polymerization Reactions using the divergence criterion", Process Saf. Environ., vol. 82 (B1), pp. 18-25, 2004.

[9] J. Bosch, F. Strozzi, J. P. Zbilut, and J. M. Zaldívar, "On line application of the divergence criterion for runaway detection in isoperibolic batch reactors: Simulated and experimental results", Comput. Chem. Eng., vol. 28, pp.527-544, 2004.

[10] J. M. Zaldívar, J. Bosch, F. Strozzi, and J. P. Zbilut, "Early warning detection of runaway initiation using non-linear approaches", Communications in Nonlinear Science and Numerical Simulation, vol. 10, pp. 299-311, 2005.

[11] J. Bosch, F. Strozzi, T. J. Snee, J. A. Hare, and J. M. Zaldívar, "A comparative analysis between temperature and pressure measurements for early detection of runaway initiation", J. Loss Prev. Process Ind., vol. 17, pp. 389-395, 2004.

[12] J. Bosch, D. Kerr, T. J. Snee, F. Strozzi, and J. M. Zaldívar, "Runaway detection in a pilot plant facility", Ind. Eng. Chem. Res., vol. 43, pp. 7019-7024, 2004.

[13] J. M. Zaldívar, J. Bosch, F. Strozzi, and T. J. Snee, "Early warning detection of runaway initiation using chaos-like features", in Proccedings of the $11^{\text {th }}$ International Symposium on Loss Prevention and Safety Promotion in the Process Industries, 2004, pp. 4178-4186.

[14] J. M. Zaldívar, F. Strozzi, and J. Bosch, "The divergence as a goal function for control and on-line optimization", AIChE J., vol. 51, pp. 678-681, 2005

[15] V. I. Arnold, Ordinary differential equations, Cambridge, MIT Press, 1973.

[16] J. M. Zaldívar, H. Hernández, and C. Barcons, "Development of a mathematical model and a simulator for the analysis and optimisation of batch reactors: Experimental model characterisation using a reaction calorimeter", Thermochim. Acta, vol. 289, pp. 267-302, 1996.

[17] J. M. Zaldívar, H. Hernández, H. Nieman, E. Molga, and C. Bassani, "The FIRES project: experimental study of thermal runaway due to agitation problems during toluene nitration", J. Loss Prev. Process Ind., vol. 6, pp. 319-326, 1993.

[18] N. Packard, J. Crutchfield, D. Farmer, and R. Shaw, "Geometry from a time series", Phys. Rev. Lett., vol. 45: 712-715., 1980.

[19] F. Takens "Detecting strange attractors in fluid turbulence", in Dynamical systems and Turbulence, Berlin: Springer, 1981, pp. 366381.

[20] J. P. Zbilut, J. M. Zaldívar, and F. Strozzi, "Recurrence quantification based-liapunov exponents for monitoring divergence in experimental data", Phys. Lett. A, vol. 297, pp. 173-181, 2002.

[21] T. Schreiber, and E. J. Kostelich, "Noise reduction in chaotic time series data: A survey of common methods", Phys. Rev. E, vol. 48, pp. 1752-1763, 1993

[22] T. Schreiber, and M. Ritcher,"Fast nonlinear projective filtering in a data stream", Int. J. Bifur. Chaos, vol. 9, pp. 2039-2045, 1999. 